\title{
Integrative Model of the Relationship Between Sleep Problems and Risk for Youth Substance Use
}

\author{
Sarah Edwards $^{1}$ - Gloria M. Reeves ${ }^{1} \cdot$ Diana Fishbein $^{2}$
}

Published online: 3 April 2015

(C) Springer International Publishing AG 2015

\begin{abstract}
A burgeoning literature implicates sleep problems in risk for adolescent substance misuse as well as a negative prognostic indicator of substance abuse treatment response. Mechanisms underlying the relationship between sleep problems and propensity to abuse substances during this phase of development have yet to be elucidated. Many questions also remain about this relationship given the paucity of integrative models and data from prospective studies with a pre-drug exposure baseline. Our integrative, temporal model theorizes that sleep problems may increase propensity to substance misuse through interactions with emotion dysregulation and cognitive deficits. Stress exposure may exacerbate this confluence of factors, and ongoing hormonal and brain changes during puberty may also contribute to the relationship between stress and risk for substance misuse. Finally, substance use itself worsens sleep problems and further dysregulates emotion and cognition, promoting an escalating pattern of use. If sleep
\end{abstract}

This article is part of the Topical Collection on Adolescent Substance Abuse

Diana Fishbein

Dfishbei@psych.umaryland.edu

Sarah Edwards

sedwards@psych.umaryland.edu

Gloria M. Reeves

greeves@psych.umaryland.edu

1 Division of Child and Adolescent Psychiatry, Department of Psychiatry, School of Medicine, University of Maryland, $701 \mathrm{~W}$ Pratt Street, Baltimore, MD 21201, USA

2 Center for Translational Research on Adversity, Neurodevelopment and Substance Abuse, Department of Psychiatry, School of Medicine, University of Maryland, 5900 Waterloo Road, 2nd floor, Ellicott City, MD 21045, USA problems increase risk for substance misuse and treatment intractability, greater attention to causes of sleep problems in young people would have significant preventive and/or ameliorative implications given the malleability of these functions.

Keywords Sleep problems $\cdot$ Adolescence $\cdot$ Substance misuse $\cdot$ Emotional dysregulation $\cdot$ Executive cognitive function $\cdot$ Stress

\section{Introduction}

Numerous recent reports suggest that sleep problems from adolescence into adulthood are predictive of substance use (illicit drugs and alcohol) initiation and escalation [1-3], and yet the mechanisms underlying this relationship have not been fully elucidated. Studies show that adolescent and adult substance users report more frequent sleep problems than nonusers, both prior to and after initial drug use $[2,3,4 \cdot \bullet$, 5]. For example, abstinence in chronic marijuana users is typified by dysregulation of sleep patterns that increases risk for relapse and interferes with treatment $[6,7]$. There is also some evidence that children and adolescents with particular sleep problems (over and above those that onset with puberty and characterize adolescence) are more prone to eventually initiate substance use [1-3]. Many questions remain about this putative relationship given that prospective studies with a pre-drug exposure baseline are few and integrative models are lacking.

A systems approach [8] is needed that examines the complex linkages between sleep disturbances and substance misuse, taking into account the array of biological and experiential factors (e.g., emotional regulatory and executive functioning, stress exposure, and physiologic stress response) [9-12] that may either explain or exacerbate the relationship. Reciprocal associations have been reported between these 
individual-level factors and sleep problems [2, 3, 13, 14]. Sex and pubertal changes also need to be taken into account based on findings that boys and girls differ in sleep patterns and the extent to which sleep is associated with substance use [14], and that pubertal development is typified by dramatic changes in sleep, stress reactivity, and emotion regulation [15]. Thus, a more comprehensive conceptual model that accounts for these multiple potentially influential factors could shed light on each of their roles in alcohol and illicit drug abuse to more effectively target preventive and treatment interventions. Evidence to support the idea that sleep problems in childhood may increase the probability of substance use onset, escalation, and treatment intractability would highlight the importance of preventing or ameliorating sleep problems among youth, given the long-term implications of sleep problems and substance use on health. This article reviews the pertinent literature on the relationship between sleep problems and substance involvement, as well as other factors at play during adolescence reported to influence both sleep patterns and substance use. An integrative systems model is then introduced, which proposes ways in which these influential factors may underlie or exacerbate the link between sleep and substance involvement in adolescence.

\section{Prevalence of Sleep Problems in Adolescence}

Sleep is a restorative function that is essential for healthy development and brain maturation. It is central to processing of information, learning, and memory consolidation [16]. Important changes in sleep occur during adolescence, particularly with the onset of puberty but also due to changing social demands. National survey data indicate normative age variation in sleep duration from adolescence through early adulthood, such that sleep duration decreased from $8.5 \mathrm{~h}$ nightly at age 13 years to $7.3 \mathrm{~h}$ at age 18 years [17]. Along with a decrease in sleep duration in adolescence, there is a delay in bedtime, a shift to an evening chronotype (i.e., preference for evening activities), and a decrease in the total sleep duration $[18,19]$. These changes are accompanied by a decrease in the arousal threshold and a decrease in slow-wave sleep due to maturational changes in neuronal connectivity in adolescence $[18,19]$. Normative developmental changes in adolescent sleep patterns are well documented and exist across numerous countries and cultures; worldwide, adolescents have delayed bedtimes in general and later wake times on weekends [20].

Although the National Sleep Foundation recommends 8.5$9 \mathrm{~h}$ of sleep for adolescents [21], parents reported $58 \%$ of 15 17 -year olds sleep $7 \mathrm{~h}$ or less per night and only $10 \%$ sleep $9 \mathrm{~h}$ or more [22]. These data are consistent with the Center for Disease Control and Prevention's 2013 Youth Risk Behavioral Surveillance (YRBS) data which showed $68.6 \%$ of students in 9-12th grade averaged less than $7 \mathrm{~h}$ of sleep on school nights [23]. Recognizing the importance of sleep, the US Department of Health and Human Services made it a Healthy People 2020 goal to have $33.1 \%$ of students in 9-12th grade obtain sufficient sleep [24]. Despite this increased awareness, no significant increases/trends in sleep duration were identified in the 2007-2013 YRBS data, and the Healthy People goal has yet to be reached [23]. A systematic review on adolescent sleep duration described a decrease in sleep duration of $0.75 \mathrm{~min}$ per year in children and adolescents over the past 100 years [25], suggesting shorter sleep duration for each successive generation of youth in the past century.

\section{Sleep Problems and Propensity for Substance Misuse in Adolescence}

Insufficient sleep and/or misaligned sleep patterns are known to have adverse physical and mental health outcomes. Sleep deprivation has been linked to obesity [26, 27], mood problems, and suicidal ideation [28-31]; academic and school concerns [13, 18, 32•]; and conduct problems [33]. Inadequate sleep and other sleep problems are further associated with earlier onset and increased use of alcohol, alcohol-related problems, tobacco, and other substance use [4••, 34-37]. A cross-sectional study examined data from the 2007 YRBS ( $n=$ 12,000 9-12th graders across the USA) and found that adolescents who reported sleeping less than $8 \mathrm{~h}$ on school nights reported more health risk behaviors, such as substance use (the prevalence of cigarette, marijuana, and alcohol use was $24,23.3$, and $50.3 \%$, respectively, among youth with insufficient sleep) [38]. Differences in sleep architecture found in youth at high risk for substance use disorders (alcohol and illicit drugs) based on family history compared to those at low risk $[18,39-41]$ also suggest that sleep problems may predate and possibly contribute to substance misuse.

The extent of sleep deprivation may be associated with likelihood of engaging in risk behaviors. One study found that the relationship between certain high-risk behaviors among those with inadequate sleep was non-linear and varied with the degree of sleep deprivation in the 2011 YRBS [42]. Specifically, 9th-12th grade students getting $5 \mathrm{~h}$ of sleep were $52 \%$ more likely to report smoking relative to students getting $8 \mathrm{~h}$ of sleep; students getting less than $5 \mathrm{~h}$ of sleep were $159 \%$ more likely to report smoking [42]. Another investigation of gradations of sleep deprivation examined the difference an hour or two more sleep makes for adolescent risk behaviors [43]. This study evaluated 8th, 10th, and 12th graders in a suburban community with early high school start times (e.g., 7:20 am). Students reported only $6.5 \mathrm{~h}$ of weekday sleep, which is significantly less sleep than high school students nationally. Substance use increased steadily with decreasing sleep duration in a linear manner. After controlling for background variables, the odds of a student recently using tobacco, 
alcohol, and/or marijuana increased by $23 \%$, and the odds of using illicit/prescription drugs increased by $37 \%$ for each hour less of sleep.

Pasch et al. examined longitudinal bi-directional relationships between sleep duration and patterns of substance use in a 2-year follow-up study of 704 US adolescents. Results showed that reduced weekday sleep duration was associated with increased alcohol use at baseline and shorter weekend oversleep at the 2-year follow-up. Cigarette smoking and weekend sleep were bi-directionally related as were marijuana use and total sleep, providing further evidence for causal relationships between sleep and substance use among adolescents [44].

These studies highlight the association between substance use and sleep problems in adolescence. A comprehensive, systems-based, and developmental approach is needed to understand the various factors that mediate and moderate this relationship. This work can serve to identify novel targets and strategies for primary prevention, treatment, and relapse prevention.

\section{Factors Implicated in the Relationship Between Sleep and Substance Misuse in Adolescents}

The following sections review literature on several factors which have been independently identified as possible mediators and moderators of the association between sleep problems and substance involvement in adolescence: neurocognition, emotion dysregulation, stress exposure, pubertal development, and sex. Based on associations reported between these factors and both sleep problems and substance misuse during adolescence as well as their inherent interrelatedness, an integrated systems science model is presented that postulates a specific role for each factor.

\section{Neurocognitive Mechanisms Underlying the Influence of Sleep Problems on Substance Misuse}

Neurocognition refers to cognitive functions that have been associated with activity in particular regions of the brain. Of relevance here are dimensions of executive cognitive functioning (ECF), higher-order cognitive functions, modulated by the prefrontal cortex, that involve complex planning, problem solving, decision-making, working memory, and selfregulation of goal-directed behaviors [45]. There is accumulating evidence that the relationship between inadequate or problematic sleep and substance use is related to the negative effect of sleep loss and/or irregular sleep patterns on ECF [1]. Research in adults suggests that poor sleep - acute and chronic - has neurocognitive consequences such as impaired attention and vigilance [46] as well as altered decision value signals [47] and reduced functional connectivity between affect and regulatory regions in the limbic system [48]. Both chronic and brief periods of sleep deprivation have been associated with changes in metabolism to the prefrontal region in both adolescents and adults [49•], which may underlie impairments in neurocognition. Sleep loss and poor sleep quality also have been implicated in other behaviors associated with ECF deficits such as risk-taking behaviors $[49 \bullet, 50 \bullet \bullet]$, low self-control $\left[51^{\bullet}\right]$, and delinquent behaviors $\left[51 \bullet, 52,53^{\bullet}\right]$ Since the developing adolescent brain is characterized by the imbalance of increased reward-seeking behaviors and immature impulse control (Dual Systems Model) [54, 55], the detrimental effects of poor sleep on an individual's propensity to engage in risky behaviors may be amplified during adolescence.

Results from a recent neuroimaging study support the notion that poor sleep quality is associated with greater likelihood to engage in risky behavior through increased sensitivity to reward and lower inhibitory control. Specifically, Telzer and colleagues used functional magnetic resonance imaging (fMRI) to examine brain activity associated with cognitive control and reward processing during risk-taking tasks in healthy adolescents. Adolescents who reported poorer sleep quality exhibited greater risk taking which corresponded with less dorsolateral prefrontal cortex (DLPFC) activation during cognitive control (impaired regulatory control). Lower sleep quality also showed increased insula activation during reward processing and reduced functional coupling between the DLPFC and the affective regions during reward processing (increased reward sensitivity) [50••]. These results suggest impairment in cognitive and behavioral regulation is associated with sleep quality in adolescents, and such impairments have been repeatedly related to risk for substance misuse $[9$, $56,57]$.

In addition to the effects of sleep loss on ECF, circadian misalignment is associated with impaired affective and motivational regulation [58-60, 61••]. Circadian misalignment is the discrepancy between one's internal circadian timing and imposed early school/work schedules which causes sleep loss during the week and increased sleep during the weekends; this significant difference in weekday and weekend sleep timing produces a "social jetlag" $[62,63]$. Extant research shows that the reward system is modulated by circadian rhythms and sleep loss [48, 64-66]. Hasler and colleagues reported that differences in weekday-weekend sleep timing in healthy adolescents were associated with decreased activation in the medial prefrontal cortex and ventral striatum during the anticipation and receipt of reward [60]. Based on the described research findings, Hasler and Clark proposed a conceptual model whereby circadian misalignment and reward function serve as a pathway to adolescent substance use [63, 67]. The combination of normative developmental changes (preferred eveningness) and early school start times cause many adolescents to have a chronic circadian misalignment (weekday sleep loss and social jet lag) which can lead to reward 
dysfunction (reduced regulatory control and increased sensation seeking) and risk for alcohol use.

\section{Emotional Regulatory Deficits Implicated in the Relationship Between Sleep Problems and Substance Misuse During Adolescence}

ECF and the ability to regulate emotion are integrally linked; prefrontal cognitive functions modulate emotional responses of the limbic system, contributing to decision-making within a social context $[68,69]$. Deficits in ECF may affect emotional responses via a reduction in inhibitory controls and/or inaccurate appraisals of environmental or interpersonal inputs. Dysfunction of these emotional centers within this neural network may play a direct, instrumental role in behavioral problems by compromising the ability to regulate reactions to such inputs. Studies using cognitive tasks and biological measures that activate this circuitry show that a combination of propensity to negative affect and inability to shift behavioral strategies in response to anticipated consequences is associated with substance misuse [70, 71]. Of further relevance to an understanding of the sleep-drug use relationship, sleep impairments have been typified by several indices reflective of dysregulated affective responses. Sleep problems have been shown to disrupt emotional processes involving the amygdala and prefrontal cortex [72-75]. To date, research findings have addressed direct relationships between sleep, and ECF and emotion regulation. Given the high degree of interrelatedness between ECF and emotion regulation, and their mutual association with both sleep problems and substance misuse, it is likely that these factors operate as a system.

In studies of the acute effects of sleep deprivation, controlled adolescent sleep restriction influences on emotion regulation and ECF has been studied in experimental human and animal studies. A paper reviewing 13 pediatric experimental sleep restriction studies reported that the majority of studies found sleep restriction significantly and negatively influenced neurobehavioral functioning, with a particular effect on attention and emotional lability outcomes [76]. Mixed findings across studies for other specific neurobehavioral outcomes were largely attributed to small sample sizes and design differences (e.g., single night versus chronic sleep restriction, task performance versus self-report outcomes). The authors of the review further recommended that sleep restriction studies should be structured relative to the youth's baseline sleep (e.g., $25 \%$ decrease in sleep time rather than a set sleep duration) to ensure that the restriction is a change from typical sleep.

Two other recent experimental studies of sleep restriction in adolescents provide further support for effects of sleep restriction on emotion dysregulation [77] and reduced pedestrian safety behaviors [78•]. Regarding sleep effects on emotion regulation, a sleep manipulation study assigned healthy adolescents $(n=50)$ to 5 days of sleep restricted $(6.5 \mathrm{~h})$ and healthy sleep duration $(10 \mathrm{~h})$ in a randomized order, crossover design [77]. The sleep restriction condition was associated with more irritability and worse emotional regulation compared to the healthy sleep condition. Results were consistent across both adolescent and parent report, and occurred over just a few days of restricted sleep. In another study, which used a similar crossover design, the impact of sleep restriction on pedestrian safety behaviors in a sample of healthy 14-15year olds $(n=55)$ was assessed in a virtual reality lab [78 $]$. Youth experienced more virtual motor vehicle "hits" or close calls as well as decreased time between street crossing and before virtual contact with vehicles after restricted sleep (4 h) compared to performance after adequate sleep $(8.5 \mathrm{~h})$. These high-risk behaviors related to pedestrian safety reflected difficulties with impulse control and rapid/precise decisionmaking associated with sleep restriction. Significant differences were noted after only one night of sleep restriction and results were maintained after controlling for average total sleep duration before each condition. Even brief sleep deprivation of healthy adolescents can adversely impact both emotion regulation and $\mathrm{ECF}$.

In addition to experimental sleep deprivation studies, literature on chronic insomnia may provide a more comprehensive, real-life, reflection of the effects of prolonged sleep reduction and sleep debt on cognitive and emotional regulatory functions that are often implicated in risk for substance misuse. In a prospective study following a large community sample of adolescents over 1 year, youth with chronic insomnia (defined by DSM-IV criteria) reported more negative emotional effects, such as problems with interpersonal relationships [79]. Ward et al. compared young and older adults to isolate the effects of chronic sleep restriction from aging using magnetic resonance imaging (MRI). Results indicated that connectivity within the default mode network (DMN) is reduced during sleep and associated with poor memory performance and is impaired in younger individuals who are sleep deprived [80]. Another MRI study found reduced gray matter in the ventromedial prefrontal and orbitofrontal cortices, corresponding with poorer neurobehavioral performance, in adults with sleep disturbances [81]. Oginska et al. also found detrimental effects of reduced sleep length on mood in adolescents [82]. In partial contrast, Friedman et al. reported that sleep problems in early childhood did not predict later executive functioning; however, a decrease in sleep problems over time was associated with better general executive control in late adolescence [83]. In summary, although many questions remain unaddressed, the aforementioned studies suggest both short and chronic sleep deprivation affect reactivity and regulation of emotion and ECF [76, 77, 78•, 79-84], and are predictive ofand associated with-substance misuse. 


\section{Other Potential Exacerbators of the Sleep-Substance Misuse Relationship}

\section{Stress Exposures and Physiological Responses to Stress}

In addition to the biological changes that occur in adolescence, chronic physical and psychosocial stress (e.g., family dysfunction, poverty, child maltreatment, trauma) can also impact sleep and place youth at risk for sleep disturbances. The focus here is on chronic, not necessarily acute or severe, stress based on previous research that the effects of trauma on drug use risk appear to be established earlier in childhood (ages 10-14 or younger) and persist in a relatively stable manner into middle adolescence when the risk for drug use may be heightened [85]. Stress activates the hypothalamic-pituitary-adrenal axis (HPA) which plays an essential role in sleep-wake cycles and functioning of the circadian system [86], as well as emotion regulation [87]. Both human and animal studies show a bidirectional relationship between the regulation of the HPA axis and sleep variations [88]. Typical sleep architecture changes caused by stress include decreases in sleep efficiency and increases in awakenings [86, 89]. For example, stress can cause glucocorticoid receptors in the amygdala to become preferentially activated which increases cortisol-releasing hormone, which in turn decreases slow-wave sleep and results increased wakefulness [90] (for a complete review of the interactions of the HPA axis and sleep, see Buckley and Schatzberg [88]). Prolonged activation of this system due to chronic stress can stimulate an overproduction of glucocorticoids, which may reflect a dysregulated response to stress, increasing risk for sleep disruptions [91, 92], attentional and inhibitory dyscontrol [93], poor social competence [94], cognitive deficits [95], and drug abuse [96]. Dysfunction of the neuroendocrine regulation of sleep due to stress, and the emotion dysregulation that often results, may be contributing factors to a significant subgroup of adolescents that experiences inadequate sleep.

\section{Pubertal Development, Sex Differences, and Circadian Misalignment}

Adolescent delayed sleep onset may be influenced by biological alterations in homeostatic and circadian regulatory systems that occur normally during puberty [15, 97-99]. Pubertal changes contributing to phase delay include a later timing of melatonin secretion (a biological marker of the internal circadian clock) as well as a less robust homeostatic response to sleep deprivation [15, 97-99]. Hagenauer and colleagues described similar pubertal changes in other mammalian species lending support that a juvenile phase delay is biologically based and not solely the result of psychosocial factors [97]. A lengthening of the intrinsic period of the circadian clock as well as a possible heightened sensitivity to evening light are circadian system changes which may impact the phase delay $[15,98]$. The union between changing biological sleep patterns and external factors such as early school start times and academic/social pressures may cause a circadian misalignment leading to inadequate sleep in adolescence $[61 \bullet \bullet, 98$, 99], further exacerbating the link between sleep problems and substance misuse in adolescence. Relatedly, there is additional evidence that youth with earlier pubertal maturation may experience greater problems with sleep compared to youth who are not as developed [100] and that earlier pubertal maturation has also been related to risk behavior [101-103].

Another factor that should be taken into account when studying sleep, stress, pubertal development, and substance use is sex; a myriad of studies report differences between girls and boys in each of these domains. Boys tend to exhibit an earlier onset of drug use than girls and maintain higher levels of use until about age 21 [104]. In a prospective study, sleep problems were predictive of drug use onset in males but not females, controlling for internalizing and externalizing symptoms [14]. One plausible explanation for both observations is sex differences in HPA functions and their timing $[105,106]$. Basal levels of cortisol, for example, differ between sexes; Klimes-Dougan et al. reported higher salivary cortisol levels in females than males at mid- and late afternoon [107]. Gonadal steroids are known to interact with the HPA axis, and estrogens have been shown to stimulate HPA axis activity [108-110]. Furthermore, recent studies indicate that exposure to stressors during pubertal maturation may result in enduring changes in HPA responsiveness in adulthood that differ between the sexes [111]. These hormonal processes have been related to sleep, emotion regulation, and early-onset drug use [112].

On the other hand, pubertal development and its timing have been associated with insomnia in girls [113-115]. Johnson et al. found that following the start of menses, girls had more than twice the risk of insomnia than boys. Menstruation was specifically related to problems with nonrestorative sleep and maintaining sleep. Interestingly, the higher rates of insomnia in girls were not explained by higher rates of depression [114]. This finding was recently supported by a populationbased study looking at the prevalence of insomnia symptoms in young and preadolescent children using subjective and objective sleep measures. The study found that insomnia, measured by polysomnograph sleep disturbances, peaked in girls ages 11-12 years and was related to changes associated with the onset of puberty rather than depression or anxiety [116]. Although there are contrasting literatures with respect to the role of sex in the sleep-substance use relationship, the takeaway messages may be that (a) normative changes during puberty have potential to disrupt sleep patterns, (b) girls who experience insomnia with onset of menses may be at greater risk for substance misuse, and (c) boys may be at greater risk for substance misuse in part as a function of lower HPA 
diurnal activity. Important to note, though, is that other factors, such as parental supervision, minimizing contact with deviant peers, help to constrain risk for most youth, who may experiment, but not progress to regular, heavy substance use.

\section{Integrative Model of Mechanisms Underlying Sleep Problems and Substance Involvement in Adolescents}

The extant literature leads us to conclude that many dynamic factors likely play a role in the relationship between insufficient or misaligned sleep (e.g., due to phase delay) and substance use initiation and escalation of use in adolescence. Accordingly, we developed an integrative, temporal model (see Fig. 1) hypothesizing that certain of these factors mediate (i.e., help to explain) this relationship while other factors act as moderators and, thus, explain subgroup differences (e.g., sex, extent of exposure and reaction to stress, stage or status of pubertal maturation) in outcome. The model theorizes that the relationship is partially but significantly mediated by dysregulation of interrelated cognitive and affective systems, as reflected in neuroimaging paradigms that involve performance on tasks that recruit specific prefrontal and limbic structures. These intrinsically connected neural networks are both affected by sleep problems and associated with risk for substance use and maintenance/escalation of subsequent use [3]. Exposure to prolonged stress (e.g., maltreatment, divorce, poverty) is expected to amplify this mechanistic relationship based on evidence that it disrupts sleep, induces cognitive and

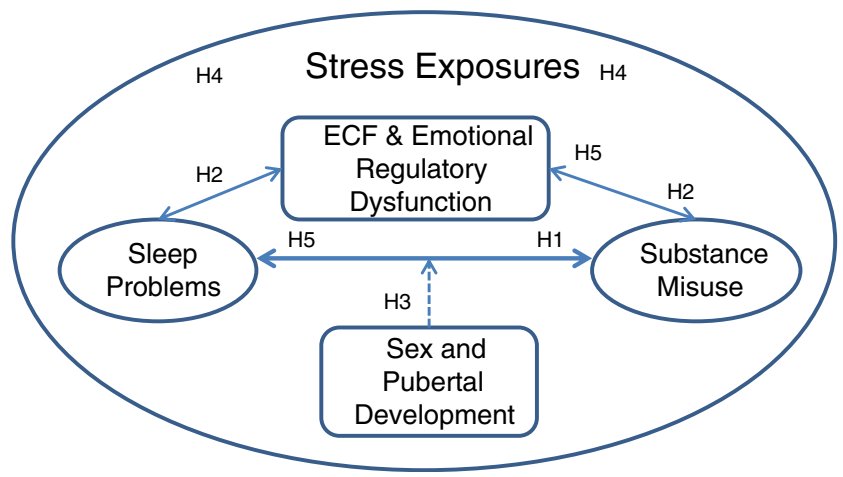

Fig. 1 Hypotheses are as follows: $H 1$ Severity of problems with sleep (e.g., hours of sleep, quality of sleep, delayed sleep phase, next day functioning) will predict initiation and maintenance of substance use in adolescence; $H 2$ the relationship between sleep problems and substance misuse will be partially mediated by level of ECF (e.g., sustained attention, memory, mental flexibility, planning, impulse control, and processing speed) and emotion regulation, as reflected in performance on tasks that recruit prefrontal and limbic structures and brain activation patterns, survey measures, and physiological measures of stress reactivity; $H 3$ sex and pubertal development will moderate the relationship between sleep problems and substance misuse; $H 4$ lifetime stress exposures will be associated with sleep problems and cognitive and emotion dysregulation to, in turn, amplify their effects on substance use onset and escalation; and $H 5$ subsequent substance use will be associated with further decrements in sleep, cognitive functioning, emotion regulation, and poor stress reactivity, leading to an escalating pattern of use emotional dysregulation, perturbs HPA functioning (e.g., cortisol, alpha amylase), and directly influences risk for substance involvement [91-96]. Sex and pubertal development are also key players in this model given findings that sleep problems are more predictive of substance use initiation in boys than in girls [14], and that pubertal development is related to poorer sleep quality and duration which is in turn is associated with increases in reward/sensation seeking, alterations in HPA function, and onset of psychological disorders at onset of puberty $[15,96,105,106,111,116,117]$. Although the proposed model applies to many drugs of abuse, it has particular import for tobacco, marijuana, and alcohol use given their prominence as the drugs most commonly initiated and used during adolescence (Fig. 1).

Our model also predicts that eventual substance use (i.e., cycles of use and cessation) will exacerbate sleep problems and lead to further decrements in emotion regulation and neurocognition, promoting an escalating pattern of use. For example, marijuana and alcohol both produce somnolence which may reinforce their use $[34,118]$. Evidence for the effects of substance use on ECF and emotion regulation, which may exacerbate and maintain the association between sleep and substance use, is reviewed in the following section.

\section{Subsequent Effects of Substance Misuse on Underlying Mechanisms of ECF and Emotion Regulation}

When substance use progresses from experimentation to chronic use, a worsening in executive and emotional functioning has been reported; while some studies show at least partial remission with sustained abstinence [119], others do not [120]. For example, in a study of older adolescents, those with a history of persistent binge drinking over a 2-year period performed worse on tasks assessing working memory, episodic memory, and executive functioning compared to individuals with no prior or discontinued binge drinking behavior [121]. Tasks that differentiated the binge and no-binge groups were described as sensitive to functioning in temporomesial and prefrontal brain regions. These findings suggest positive effects of sustained abstinence among former drinkers. Other works indicate that total years of alcohol use and acute intoxication (BAC ranging $0-0.287$ ) in adolescents were both associated with impaired performance on a mental flexibility task. However, only greater chronicity of alcohol use was associated with impairment in visuomotor performance [122]. These results suggest that the association between duration of alcohol use and impaired motor performance can be detected before adulthood. Chronic marijuana use also has been shown to be associated with reduced anterior cingulate and cerebellar activation in response to a motor task (i.e., finger tapping) in adolescents compared to healthy controls [123]. In addition, among adolescent chronic marijuana users, spectroscopy findings implicate glutamatergic 
and GABAergic abnormalities underlying cingulate dysfunction [124].

Disentangling the precursors from the consequences of substance use represents one of the most fundamental challenges in the field of substance misuse, with the greatest implications for its prevention and treatment. Few attempts have been made so far to apply an integrated model to understand the inherent linkages and temporal dynamics between factors consistently and strongly implicated in substance use initiation and escalation. Of relevance, hundreds of studies implicate stress exposures, physiological stress hyporeactivity, and deficits in ECF and emotion regulation in propensity to substance misuse. And more recently, sleep disturbances, independently associated with each of these risk factors, have also been strongly related to substance use onset, maintenance, and escalation [2, 5, 125-127]. Adolescents experiencing sleep problems may self-medicate with substances either to increase alertness or to induce sleep [5, 128]. Studies have traditionally addressed each of these phenomena separately despite their interconnectedness. A systems science approach to this question emphasizes the highly interrelated nature of these factors; understanding substance misuse can be substantially improved by considering these factors simultaneously.

\section{Future Directions}

To increase understanding of the role of sleep in adolescent substance use initiation and advance the field, studies are needed to further define this relationship that (a) are prospective to flesh out temporal ordering of relationships, (b) more comprehensively test system-based hypotheses, (c) focus on individual-level factors, and (d) aim to identify mechanisms (e.g., cognitive skills, emotion regulation, coping) that underlie the relationship that can be altered through palatable means to redirect substance use pathways. Identifying contextual and experiential conditions that modify this relationship will lead to the design of interventions that account for conditions that promote and, alternatively, interfere with successful outcomes.

The implications of this line of research are substantial, particularly in light of reports that at least $25 \%$ of children and adolescents experience sleep problems at some point $[14$, 129], and according to the National Sleep Foundation (www. sleepfoundation.org), a significant proportion of teenagers are at least somewhat sleep deprived and impaired as a result. Ultimately, sleep problems (e.g., long latency to sleep, deprivation, disruptions) may exacerbate the tendency toward reward-driven behavior in adolescence [130] by impacting neural networks subserving emotion regulation. "Because decreased sleep has been associated with risky behavior, negative mood and cognitive deficits, these findings raise concerns about a negative spiral whereby the effects of puberty and sleep deprivation may have synergistic effects on reward processing, contributing to adolescent behavioral and emotional health problems" [116] such as substance misuse. In the absence of intervention, however, the co-occurrence of these commonly associated factors may contribute to addictive vulnerability across the life span. Fleshing out these relationships will help advance the field in terms of its prominence and placement in the relationship between stress exposure; behavioral, physiological, and cognitive processes; sex; and maturational differences in these developmental trajectories.

Moreover, understanding how sleep problems may promote drug use and escalation could provide insights not only into the process of addiction but also into the functioning of neurotransmitter systems and HPA systems since they play a modulatory role in sleep. Importantly, sleep problems are more often than not remediable, and the factors proposed to mediate and moderate this relationship are to a great extent malleable especially if identified early [131]. Elucidating the role of and underlying mechanisms in sleep disturbance in children at risk and active substance users has potential implications for understanding onset and relapse, and guiding treatment protocols. For example, sleep disturbance is a potential target for the management of drug use disorders since poor sleep could contribute to relapse and treatment failure in drug users [132]. A multitude of treatment options exist for sleep problems, contingent on the type (e.g., insomnia, periodic leg movements, apnea, period limb movement, REM disorders) and source (e.g., stress, sleep hygiene, environment, hormone disruption, caffeine) that can be clinically determined. Britton et al. reported that a multicomponent behavioral sleep intervention for drug-abusing adolescents improved sleep which was, in turn, associated with reductions in drug use-related problems. Intervening before puberty-between the ages of about 9 and 13-is a critical time to address the problem, prior to the dramatic changes that typically occur in adolescence, including the spike in rates of psychological, emotional, and behavioral disorders and when the brain is highly plastic [133]. These trajectories differ between the sexes in ways that are clinically pertinent. This research has further implications for educating parents and youth about more adaptive sleep hygiene habits, and consideration of policies to delay start times for high schools may also help teens to better regulate their sleep cycles. Thus, this information has great preventive value and could further reduce later dependency on prescription and illicit drugs to temporarily ameliorate the sleep disturbance.

\section{Compliance with Ethics Guidelines}

Conflict of Interest Sarah Edwards, Gloria Reeves, and Diana Fishbein declare that they have no conflict of interest.

Human and Animal Rights and Informed Consent This article does not contain any studies with human or animal subjects performed by any of the authors. 


\section{References}

Papers of particular interest, published recently, have been highlighted as:

- Of importance

•- Of major importance

1. Conroy DA, Arnedt JT. Sleep and substance use disorders: an update. Curr Psychiatry Rep. 2014;16:487.

2. Wong MM, Brower KJ, Fitzgerald HE, Zucker RA. Sleep problems in early childhood and early onset of alcohol and other drug use in adolescence. Alcohol Clin Exp Res. 2004;28(4):578-87.

3. Wong MM, Brower KJ, Nigg JT, Zucker RA. Childhood sleep problems, response inhibition, and alcohol and drug outcomes in adolescence and young adulthood. Alcohol Clin Exp Res. 2010;34(6):1033-44.

4.• Hasler BP, Martin CS, Wood DS, Rosario B, Clark DB. A longitudinal study of insomnia and other sleep complaints in adolescents with and without alcohol use disorders. Alcoholism: Clin Exp Res. 2014;38:2225-33. In a longitudinal study investigating adolescents with and without alcohol substance use disorder (AUD), youth with AUD had chronic sleep problems from baseline through 5 years. In youth without AUD at baseline, weekday-weekend differences in amount of sleep and insomnia predicted increased alcohol symptoms at follow-up.

5. Breslau N, Roth T, Rosenthal L, Andreski P. Sleep disturbance and psychiatric disorders: a longitudinal epidemiological study of young adults. Biol Psychiatry. 1996;39(6):411-8.

6. Bolla KI, Lesage SR, Gamaldo CR, Neubauer DN, Funderburk FR, Cadet JL, et al. Sleep disturbance in heavy marijuana users. SLEEP. 2008;31(6):901-8.

7. Kouri EM, Pope HG. Abstinence symptoms during withdrawal from chronic marijuana abuse. Exp Clin Psychopharmacol. 2000;8:483-92.

8. Smith LB, Thelen EE. A dynamic systems approach to development: applications. In This book grew out of a workshop, "Dynamic Systems in Development," held for the Society for Research in Child Development in Kansas City, KS, Apr 1989. The MIT Press; 1993.

9. Giancola PR, Tarter RE. Executive cognitive functioning and risk for substance abuse. Psychol Sci. 1999;10:203-5.

10. Deckel AW, Hesselbrock V, Bauer L. Relationship between alcohol-related expectancies and anterior brain functioning in young men at risk for developing alcoholism. Alcohol Clin Exp Res. 1995;19:476-81.

11. Giancola PR, Martin CS, Tarter RE, Pelham WE, Moss HB. Executive cognitive functioning and aggressive behavior in preadolescent boys at high risk for substance abuse/dependence. $\mathrm{J}$ Stud Alcohol. 1996;57:352-9.

12. Sinha R. How does stress increase risk of drug abuse and relapse? Psychopharmacology. 2001;158(4):343-59.

13. Beebe DW. Cognitive, behavioral, and functional consequences of inadequate sleep in children and adolescents. Ped Clin N Am. 2011;58:649-65.

14. Wong MM, Brower KJ, Zucker RA. Childhood sleep problems, early onset of substance use and behavioral problems in adolescence. Sleep Med. 2009;10(7):787-96.

15. Carskadon M. Sleep in adolescents: the perfect storm. Pediatr Clin N Am. 2011;58:637-47.

16. Born J, Wilhelm I. System consolidation of memory during sleep. Psychol Res. 2012;76:192-203.

17. Maslowsky J, Ozer EJ. Developmental trends in sleep duration in adolescence and young adulthood: evidence from a national United States sample. J Adoles Health. 2014;54:691-7.
18. Colrain IM, Baker FC. Changes in sleep as a function of adolescent development. Neuropsychol Rev. 2011;21:5-21.

19. Dahl RE, Lewin DS. Pathways to adolescent health sleep regulation and behavior. J Adolesc Health. 2002;31(6):17584.

20. Gradisar M, Gardner G, Dohnt H. Recent worldwide sleep patterns and problems during adolescence: a review and meta-analysis of age, region, and sleep. Sleep Med. 2011;12: $110-8$.

21. National Sleep Foundation. Teens and sleep. http:// sleepfoundation.org/sleep-topics/teens-and-sleep. Accessed December 6, 2014.

22. National Sleep Foundation. 2014 Sleep in America Poll: sleep in the modern family. Washington (DC): The Foundation; 2014. http://www.sleepfoundation.org/sleep-polls-data/sleep-in-america- poll/2014-sleep-in-the-modern-family. Accessed December 6, 2014.

23. Center for Disease Control and Prevention. Youth risk behavior surveillance-United States, 2013. Morbidity and Mortality Weekly Report. 2014;63(SS-4):42.

24. Sleep Health. Healthy People 2020 topics and objectives. http:// www.healthypeople.gov/2020/topics-objectives/topic/sleephealth/objectives. Accessed December 6, 2014.

25. Matricciani L, Olds T, Petkov J. In search of lost sleep: secular trends in the sleep time of school-aged children and adolescents. Sleep Med Rev. 2012;16:203-11.

26. Nielsen LS, Danielsen KV, Sørensen TIA. Short sleep duration as a possible cause of obesity: critical analysis of the epidemiological evidence. Obes Rev. 2011;12(2):78-92.

27. Kong AP, Wing YK, Choi KC, Li AM, Ko GT, Ma RC, et al. Associations of sleep duration with obesity and serum lipid profile in children and adolescents. Sleep Med. 2011;12(7): 659-65.

28. Sarchiapone M, Mandelli L, Carli V, Iosue M, Wasserman C, Hadlacky G, et al. Hours of sleep in adolescents and its association with anxiety, emotional concerns, and suicidal ideation. Sleep Med. 2014;15:248-54.

29. Wong MW, Brower KJ, Ukcer RA. Sleep problems, suicidal ideation, and self-harm behaviors in adolescence. J Psychiatr Res. 2001;45:505-11.

30. Touchette E, Chollet A, Galera C, Fombonne E, Falissard B, Boivin M. Prior sleep problems predict internalizing problems later in life. J Affect Disord. 2012;143:166-71.

31. Millman RP. Excessive sleepiness in adolescents and young adults: causes, consequences, and treatment strategies. Pediatrics. 2005;115(6):1774-86.

32. Asarnow LD, McGlinchey E, Harvey A. The effects of bedtime and sleep duration on academic and emotional outcomes in a nationally representative sample of adolescents. J Adoles Health. 2014;54:350-6. In a sample of $\mathbf{2 , 7 0 0}$ adolescents from the National Longitudinal Study of Adolescent Health, one third of youth with late bedtimes had greater emotional distress and worse academic performance than youth with earlier bedtimes.

33. Lin WH, Yi CC. Unhealthy sleep practices, conduct problems, and daytime functioning during adolescence. J Youth Adolescence 2014:

34. Shibley HL, Malcolm RJ, Veatch LM. Adolescents with insomnia and substance abuse: consequences and comorbidities. J Psychiatr Pract. 2008;14(3):146-53.

35. Shochat T, Cohen-Zion M, Tzischinsky O. Functional consequences of inadequate sleep in adolescents: a systematic review. Sleep Med Rev. 2014;18:75-87.

36. Kenney SR, Lac A, Labrie JW, Hummer JF, Pham A. Mental Health, sleep quality, drinking motives, and alcohol-related 
consequences: a path-analytic model. J Studies Alcohol Drugs. 2013;74:841-51.

37. Fakier N, Wild LG. Associations among sleep problems, learning difficulties, and substance use in adolescence. J Adolesc. 2011;34: 717-26.

38. Mcknight-Eily LR, Eaton DK, Lowry R, Croft JB, PresleyCantrell L, Perry GS. Relationships between hours of sleep and health-risk behaviors in US adolescent students. Prev Med. 2011;53:271-3.

39. Ehlers CL, Gilder DA, Criado JR, Caetano R. Sleep quality and alcohol-use disorders in a select population of youngadult Mexican Americans. J Stud Alcohol Drugs. 2010;71(6):879-84.

40. Tarokh L, Van Reen E, Acebo C, LeBourgeois M, Seifer R, Fallone G, et al. Adolescence and parental history of alcoholism: insights from the sleep EEG. Alcohol Clin Exp Res. 2012;9:1530 41.

41. Kirisci L, Tarter RE, Ridenour T, Reynolds M, Vanyukov M. Longitudinal modeling of transmissible risk in boys who subsequently develop cannabis use disorder. Am J Drug Alcohol Abuse. 2010;39(3):180-5.

42. Meldrum RC, Restivo E. The behavioral and health consequences of sleep deprivation among U.S. high school students: relative deprivation matters. Preventative Med. 2014;63:24-8.

43. Winsler A, Deutsch A, Vorona RD, Payne PA, Szklo-Coxe M. Sleepless in Fairfax: the difference one more hour of sleep can make for teen hopelessness, suicidal ideation, and substance use. J Youth Adolesc. 2014;1-17.

44. Pasch KE, Latimer LA, Cance JD, Moe SG, Lytle LA. Longitudinal bidirectional relationships between sleep and youth substance use. J Youth Adolesc. 2012;13.

45. Pickens S, Ostwald SK, Murphy-Pace K, Bergstrom N. Systematic review of current executive function measures in adults with and without cognitive impairments. Int J Evid Based Healthcare. 2010;8(3):110-25.

46. Lim J, Dinges DF. A meta-analysis of the impact of short-term sleep deprivation on cognitive variables. Psychol Bull. 2010;136(3):375-89.

47. Libedinsky C, Smith DV, Teng CS, Namburi P, Chen VW, Huettel SA, et al. Sleep deprivation alters valuation signals in the ventromedial prefrontal cortex. Front Behav Neurosci. $2011 ; 5$.

48. Gujar N, Yoo SS, Hu P, Walker MP. Sleep deprivation amplifies reactivity of brain reward networks, biasing the appraisal of positive emotional experiences. J Neurosci. 2011;31:4466-74.

49. Womack SD, Hook JN, Reyna SH, Ramos M. Sleep loss and risktaking behaviors: a review of the literature. Behav Sleep Med. 2013;11:343-59. In this review article, sleep loss was positively associated with risk-taking behaviors. Reduced functioning in the ventromedial prefrontal cortex was one possible mediator of the relationship between sleep loss and risk-taking behaviors.

50.• Telzer EH, Fuligni AJ, Lieberman MD, Galvan A. The effects of poor sleep on brain function and risk taking in adolescence. NeuroImage. 2013;71:275-83. In an imaging study of healthy adolescents, youth with poorer sleep quality had greater risk-taking which corresponded with decreased dorsal lateral prefrontal cortex activation (impaired regulatory control). Poorer sleep quality was associated with increased insula activation during reward processing and reduced functional coupling between the dorsal lateral prefrontal cortex and the affective regions during reward processing (increased reward sensitivity)
51. Meldrum RC, MBarnes JC, Hay C. Sleep deprivation, low selfcontrol, and delinquency: a test of the strength model of self-control. J Youth Adolescence. 2013;1-13. In a multi-site study, sleep deprivation in adolescents was positively related to low selfcontrol, and low self-control was positively related to delinquency. The article describes how the relationship between sleep deprivation and delinquency is indirect and may operate through low self-control.

52. Clinkinbeard SS, Simi P, Evans MK, Anderson AL. Sleep and delinquency: does the amount of sleep matter? J Youth Adolescence. 2011;40:916-30.

53. Peach HD, Gaultney JF. Sleep, impulse control, and sensationseeking predict delinquent behavior in adolescents, emerging adults, and adults. J of Adolescent Health. 2013;53:292-9. In a study using data from the National Longitudinal Study of Adolescent Health, sleep (duration and delayed bedtimes), impulse control, and sensation seeking predicted concurrent delinquent behavior.

54. Somerville LH, Jones RM, Casey BJ. A time of change: behavioral and neural correlates of adolescent sensitivity to appetitive and aversive environmental cues. Brain Cogn. 2010;72: 124-33.

55. Steinberg L. A dual systems model of adolescent risk-taking. Dev Psychobiol. 2010;52:216-24.

56. Tarter RE, Kirisci L, Habeych M, Reynolds M, Vanyukov M. Neurobehavior disinhibition in childhood predisposes boys to substance use disorder by young adulthood: direct and mediated etiologic pathways. Drug Alcohol Depend. 2004;73(2): 121-32.

57. Khurana A, Romer D, Betancourt LM, Brodsky NL, Giannetta JM, Hurt H. Experimentation versus progression in adolescent drug use: a test of an emerging neurobehavioral imbalance model. Development and psychopathology. 2014;1-13.

58. Pasch KE, Laska MN, Lytle LA, Moe SG. Adolescent sleep, risk behaviors, and depressive symptoms: are they linked? Am J Health Behav. 2010;34:237-48.

59. O'Brien EM, Mindell JA. Sleep and risk-taking behavior in adolescents. Behav Sleep Med. 2005;3(3):113-33.

60. Hasler BP, Smith LJ, Cousins JC, Bootzin RR. Circadian rhythms, sleep, and substance abuse. Sleep Med Rev. 2012;16(1):67-81.

61.• Hasler BP, Soehner AM, Clark DB. Circadian rhythms and risk for substance use disorders in adolescence. Curr Opin Psychiatry. 2014;27(6):460-6. This article reviews recent literature on adolescent circadian rhythms, circadian modulation of reward processes, and the associated increased risk for substance use disorders.

62. Wittmann M, Dinich J, Merrow M, Roenneberg T. Social jetlag: misalignment of biological and social time. Chronobiol Int. 2006;23(1-2):497-509.

63. Hasler BP, Clark DB. Circadian misalignment, reward-related brain function, and adolescent alcohol involvement. Alcohol Clin Exp Res. 2013;37(4):558-65.

64. Murray G, Nicholas CL, Kleiman J, Dwyer R, Carrington MJ, Allen NB, et al. Nature's clocks and human mood: the circadian system modulates reward motivation. Emotion. 2009;9(5):705.

65. Venkatraman V, Huettel SA, Chuah LY, Payne JW, Chee MW. Sleep deprivation biases the neural mechanisms underlying economic preferences. J Neurosci. 2011;31(10):3712-8.

66. Hasler BP, Sitnick SL, Shaw DS, Forbes EE. An altered neural response to reward may contribute to alcohol problems among late adolescents with an evening chronotype. Psychiatry Res Neuroimaging. 2013;214(3):357-64.

67. Hasler BP, Soehner AM, Clark DB. Sleep and circadian contributions to adolescent alcohol use disorder. Alcohol. 2014. In press 
68. Zelazo PD, Cunningham WA. Executive function: mechanisms underlying emotion regulation. 2007.

69. Banfield JF, Wyland CL, Macrae CN, Munte, TF, Heatherton TF. The cognitive neuroscience of self-regulation. Handbook of selfregulation: Research, theory, and applications, 62-83. 2004

70. Fishbein D, Hyde C, Eldreth D, London ED, Matochik J, Ernst M, et al. Cognitive performance and autonomic reactivity in abstinent drug abusers and nonusers. Exp Clin Psychopharmacol. 2005;1: 25-40.

71. Weiss F, Ciccocioppo R, Parsons LH, Katner S, Liu X, Zorrilla EP, et al. Compulsive drug-seeking behavior and relapse. Neuroadaptation, stress, and conditioning factors. Ann N Y Acad Sci. 2001;937:1-26.

72. Yoo S, Gujar N, Hu P, Jolesz F, Walker M. The human emotional brain without sleep: a prefrontal-amygdala disconnect? Curr Biol. 2007; 17:877-8.

73. Dahl RE. The impact of inadequate sleep on children's daytime cognitive function. In Seminars in pediatric neurology (Vol. 3, No. 1, pp. 44-50). WB Saunders.1996.

74. Silk JS, Vanderbilt-Adriance E, Shaw DS, Forbes EE, Whalen DJ, Ryan ND, et al. Resilience among children and adolescents at risk for depression: mediation and moderation across social and neurobiological contexts. Dev Psychopathol. 2007;19(03):841-65.

75. Talbot LS, McGlinchey EL, Kaplan KA, Dahl RE, Harvey AG. Sleep deprivation in adolescents and adults: changes in affect. Emotion. 2010;10:831-41

76. Cassoff J, Bhatti JA, Gruber R. The effect of sleep restriction on neurobehavioural functioning in normally developing children and adolescents: insights from the attention behaviour and sleep laboratory. Pathol Biol (Paris). 2014;62(5):319-31.

77. Baum KT, Desai A, Field J, Miller LE, Rausch J, Beebe DW. Sleep restriction worsens mood and emotion regulation in adolescents. J Child Psychol Psychiatry. 2014;55(2):180-90.

78. Davis AL, Avis KT, Schwebel DC. The effects of acute sleep restriction on adolescents' pedestrian safety in a virtual environment. Adolesc Health. 2013;53(6):785-90. In a sample of fiftyfive 14- and 15-year olds using a within-subjects design, the impact of sleep restriction on pedestrian safety behaviors was assessed in a virtual reality lab. Youth experienced more virtual motor vehicle "hits" after restricted sleep $(4 \mathrm{~h})$ compared to performance after adequate sleep $(8.5 \mathrm{~h})$. High safety risk behaviors reflected difficulties with impulse control and rapid/ precise decision-making.

79. Roberts RE, Roberts CR, Duong HT. Chronic insomnia and its negative consequences for health and functioning of adolescents: a 12month prospective study. J Adolesc Health. 2008;42(3):294-302.

80. Ward AM, McLaren DG, Schultz AP, Chhatwal J, Boot BP, Hedden T, et al. Daytime sleepiness is associated with decreased default mode network connectivity in both young and cognitively intact elderly subjects. Sleep. 2013;36(11):1609-15.

81. Killgore WD, Schwab ZJ, Kipman M, DelDonno SR, Weber M. Voxel-based morphometric gray matter correlates of daytime sleepiness. Neurosci Lett. 2012;518(1):10-3.

82. Oginska H, Pokorski J. Fatigue and mood correlates of sleep length in three age-social groups: school children, students, and employees. Chronobiol Int. 2006;23(6):1317-28.

83. Friedman NP, Corley RP, Hewitt JK, Wright Jr KP. Individual differences in childhood sleep problems predict later cognitive executive control. Sleep. 2009;32(3):323-33.

84. Kahn M, Sheppes G, Sadeh A. Sleep and emotions: bidirectional links and underlying mechanisms. Int J Psychophys- iol. 2013;89: 218-28.

85. Fishbein D, Novak SP, Krebs C, Warner T, Hammond J. The mediating effect of depressive symptoms on the relationship between traumatic childhood experiences and drug use initiation. Addict Behav. 2011;36(5):527-31.
86. Van Reeth O, Weibel L, Spiegel K, Leproult R, Dugovic C, Maccari S. Interactions between stress and sleep: from basic research to clinical situations. Sleep Med Rev. 2000;4(2):201-19.

87. Stansbury K, Gunnar MR. Adrenocortical activity and emotion regulation. In: Monographs of the Society for Research in Child Development, Vol. 59, No. 2/3, The Development of Emotion Regulation: Biological and Behavioral Considerations. 1994. pp. 108-134.

88. Buckley TM, Schatzberg AF. On the interactions of the hypothalamic-pituitary-adrenal (HPA) axis and sleep: normal HPA axis activity and circadian rhythm, exemplary sleep disorders. J Clin Endocrinol Metab. 2005;90(5):3106-14.

89. Kin E, Dimsdale JE. The effect of psychosocial stress on sleep: a review of polysomnographic evidence. Behav Sleep Med. 2007;5: 256-78.

90. Balbo M, Leproult R, Van Cauter E. Impact of sleep and its disturbances on hypothalamo-pituitary-adrenal axis activity. Int $\mathrm{J}$ Endocrinol. 2010;2010:759234.

91. Cicchetti D, Rogosch FA, Gunnar MR, Toth SL. The differential impacts of early physical and sexual abuse and internalizing problems on daytime cortisol rhythm in school-aged children. Child Dev. 2010;81(1):252-69.

92. Lavie P. Sleep disturbances in the wake of traumatic events. N Engl J Med. 2001;345(25):1825-32.

93. Davis EP, Bruce J, Gunnar MR. The anterior attention network: associations with temperament and neuroendocrine activity in 6year-old children. Dev Psychobiol. 2002;40:43-56.

94. Blair C, Peters R. Physiological and neurocognitive correlates of adaptive behavior in preschool among children in Head Start. Dev Neuropsychol. 2003;24(1):479-97.

95. Quas JA, Bauer A, Boyce WT. Physiological reactivity, social support, and memory in early childhood. Child Dev. 2004;75(3): 797-814.

96. Huizink AC, Ferdinand RF, Ormel J, Verhulst FC. Hypothalamicpituitary-adrenal axis activity and early onset of cannabis use. Addiction. 2006;101:1581-8.

97. Hagenauer MH, Perryman JI, Lee TM, Carskadon MA. Adolescent changes in the homeostatic and circadian regulation of sleep. Dev Neurosci. 2009;31:31276-284.

98. Jenni OG, Carskadon MA. Sleep behavior and sleep regulation from infancy through adolescence normative aspects. Sleep Med Clin. 2012;7:529-38.

99. Carskadon MA, Tarokh L. Developmental changes in sleep biology and potential effects on adolescent behavior and caffeine use. Nutr Rev. 2014;72 Suppl 1:60-4.

100. Pieters S, Van Der Vorst H, Burk WJ, Wiers RW, Engels RC. Puberty-dependent sleep regulation and alcohol use in early adolescents. Alcohol Clin Exp Res. 2010;34:1512-8.

101. Stattin H, Kerr M, Skoog T. Early pubertal timing and girls' problem behavior: integrating two hypotheses. J Youth Adolescence. 2011;40(10):1271-87.

102. Graber JA. Pubertal timing and the development of psychopathology in adolescence and beyond. Horm Behav. 2013;64(2):262-9.

103. Tremblay L, Frigon JY. Precocious puberty in adolescent girls: a biomarker of later psychosocial adjustment problems. Child Psychiatry Hum Dev. 2005;36(1):73-94.

104. Kahler CW, Read JP, Wood MD, Palfai TP. Social environmental selection as a mediator of gender, ethnic, and personality effects on college student drinking. Psychol Addict Behav. 2003;17(3):22634.

105. McEwen BS. Physiology and neurobiology of stress and adaptation: central role of the brain. Physiol Rev. 2007;87(3):873-904.

106. Netherton C, Goodyer I, Tamplin A, Herbert J. Salivary cortisol and dehydroepiandrosterone in relation to puberty and gender. Psychoneuroendocrinology. 2004;29(2):125-40. 
107. Klimes-Dougan B, Hastings PD, Granger DA, Usher BA, ZahnWaxler C. Adrenocortical activity in at-risk and normally developing adolescents: individual differences in salivary cortisol basal levels, diurnal variation, and responses to social challenges. Dev Psychopathol. 2001;13(03):695-719.

108. Burgess LH, Handa RJ. Chronic estrogen-induced alterations in adrenocorticotropin and corticosterone secretion, and glucocorticoid receptor-mediated functions in female rats. Endocrinology. 1992;131(3):1261-9.

109. Handa RJ, Burgess LH, Kerr JE, O'Keefe JA. Gonadal steroid hormone receptors and sex differences in the hypothalamopituitary-adrenal axis. Horm Behav. 1994;28(4):464-76.

110. Kiess W, Meidert A, Dressendorfer RA, Scheiver K, Kessler U, Konig A. 1995. Salivary cortisol levels throughout childhood and adolescence: relation with age, pubertal stage and weight. Pediatr Res. 1995;37:502-6.

111. Romeo RD. Pubertal maturation and programming of hypothalamic-pituitary-adrenal reactivity. Front Neuroendocrinol. 2010;31(2):232-40.

112. Camhi SL, Morgan WJ, Pernisco N, Quan SF. Factors affecting sleep disturbances in children and adolescents. Sleep Med. 2000;1(2):117-23.

113. Johnson EO, Roth T, Schultz L, Breslau N. Epidemiology of DSM-IV insomnia in adolescence: lifetime prevalence, chronicity, and an emergent gender difference. Pediatrics. 2006;117(2):e24756.

114. Knutson KL. The association between pubertal status and sleep duration and quality among a nationally representative sample of US adolescents. Am J Hum Biol. 2005;17(4):418-24.

115. Calhoun SL, Fernandez-Mendoza J, Vgontzas AN, Liao D, Bixler EO. Prevalence of insomnia symptoms in a general population sample of young children and preadolescents: gender effects. Sleep Med. 2014;15(1):91-5.

116. Holm SM, Forbes EE, Ryan ND, Phillips ML, Tarr JA, Dahl RE. Reward-related brain function and sleep in pre/early pubertal and $\mathrm{mid} /$ late pubertal adolescents. J Adolesc Health. 2009;45(4):32634.

117. Yun AJ, Bazar KA, Lee PY. Pineal attrition, loss of cognitive plasticity, and onset of puberty during the teen years: is it a modern maladaptation exposed by evolutionary displacement? Med Hypotheses. 2004;63(6):939-50.

118. Brower KJ. Alcohol's effects on sleep in alcoholics. Alcohol Res Health. 2001;25:110-25.

119. Lisdahl KM, Gilbart ER, Wright NE, Shollenbarger S. Dare to delay? The impacts of adolescent alcohol and marijuana use onset on cognition, brain structure, and function. Frontiers in Psychiatry, $2013 ; 4$.
120. Meier MH, Caspi A, Ambler A, Harrington H, Houts R, Keefe RS, et al. Persistent cannabis users show neuropsychological decline from childhood to midlife. Proc Natl Acad Sci. 2012;109(40): E2657-64.

121. Mota N, Parada M, Crego A, Doallo S, Caamaño-Isorna F, Rodríguez Holguín S, et al. Binge drinking trajectory and neuropsychological functioning among university students: a longitudinal study. Drug Alcohol Depend. 2013;133(1):108-14.

122. Day A, Celio M, Lisman S, Johansen G, Spear L. Acute and chronic effects of alcohol on trail making test performance among underage drinkers in a field setting. J Stud Alcohol Drugs. 2013;74(4):635-41.

123. Lopez-Larson M, Rogowska J, Bogorodzki P, Bueler, McGlade E, Yurgelun-Todd D. Cortico-cerebellar abnormalities in adolescents with heavy marijuana use. Psychiatry Res. 2012;202(3):224-32.

124. Prescot A, Renshaw P, Yurgelun-Todd D. $\gamma$-Amino butyric acid and glutamate abnormalities in adolescent chronic marijuana smokers. Drug Alcohol Depend. 2013;129(3):232-9.

125. Johnson EO, Breslau N. Sleep problems and substance use in adolescence. Drug Alcohol Depend. 2001;64(1):1-7.

126. Tynjälä J, Kannas L, Levälahti E. Perceived tiredness among adolescents and its association with sleep habits and use of psychoactive substances. J Sleep Res. 1997;6(3):189-98.

127. Vignau J, Bailly D, Duhamel A, Vervaecke P, Beuscart R, Collinet C. Epidemiologic study of sleep quality and troubles in French secondary school adolescents. J Adolesc Health. 1997;21(5): 343-50.

128. Carskadon MA. Patterns of sleep and sleepiness in adolescents. Pediatrician. 1990;17(1):5-12.

129. Ohayon MM, Roberts RE, Zulley J, Smirine S, Priest RG. Prevalence and patterns of problematic sleep among older adolescents. J Am Acad Child Adolesc Psych. 2000;39(12):1549-56.

130. Volkow ND, Wang GJ, Telang F, Fowler JS, Logan J, Wong C, et al. Sleep deprivation decreases binding of [11C] raclopride to dopamine D2/D3 receptors in the human brain. J Neurosci. 2008;28(34):8454-61.

131. Bootzin RR, Stevens SJ. Adolescents, substance abuse, and the treatment of insomnia and daytime sleepiness. Clin Psychol Rev. 2005;25(5):629-44.

132. Brower KJ, Perron BE. Sleep disturbance as a universal risk factor for relapse in addictions to psychoactive substances. Med Hypotheses. 2010;74(5):928-33.

133. Britton WB, Bootzin RR, Cousins JC, Hasler BP, Peck T, Shapiro SL. The contribution of mindfulness practice to a multicomponent behavioral sleep intervention following substance abuse treatment in adolescents: a treatment-development study. Subst Abus. 2010;31(2):86-97. 